\title{
Detailed Molecular Dynamics of the Photochromic Reaction of Spiropyran: A Semiclassical Dynamics Study
}

\author{
Gaohong Zhai, ${ }^{1}$ Shuai Shao, ${ }^{1}$ Shaomei Wu, ${ }^{1}$ Yibo Lei, ${ }^{1}$ and Yusheng Dou ${ }^{2}$ \\ ${ }^{1}$ Key Laboratory of Synthetic and Natural Functional Molecule Chemistry of Ministry of Education, College of Chemistry \& Materials \\ Science and Shaanxi Key Laboratory of Physico-Inorganic Chemistry, Northwest University, Xian 710069, China \\ ${ }^{2}$ Department of Physical Sciences, Nicholls State University, P.O. Box 2022, Thibodaux, LA 70310, USA
}

Correspondence should be addressed to Gaohong Zhai; zgh@nwu.edu.cn and Yibo Lei; leiyb@nwu.edu.cn

Received 10 April 2014; Accepted 20 May 2014; Published 17 June 2014

Academic Editor: Fuli Li

Copyright (C) 2014 Gaohong Zhai et al. This is an open access article distributed under the Creative Commons Attribution License, which permits unrestricted use, distribution, and reproduction in any medium, provided the original work is properly cited.

\begin{abstract}
A realistic semiclassical dynamics simulation study is reported for the photoinduced ring-opening reaction of spiropyran. The main simulation results show that one pathway involves hydrogen out-of-plane (HOOP) torsion of phenyl ring nearby $\mathrm{N}$ atom in $254 \mathrm{fs}$ on the excited state and the isomerization from cis- to trans-SP that is complete in about 10 ps on the ground state after the electron transition $\pi \sigma^{*}$; the other dominate pathway corresponds to the ring-opening reaction of trans-SP to form the most stable merocyanine (MC) product. Unlike the previous theoretical finding, one $\mathrm{C}-\mathrm{C}$ bond cleavage on the real molecule rather than the $\mathrm{C}-\mathrm{N}$ dissociation of the model one is more probable than the ring-opening reaction after the photoexcitation of SP. The simulation findings provide more important complementarity for interpreting experimental observations, confirming the previously theoretical studies of photochromic ring-opening process and even supplying other possible reaction mechanisms.
\end{abstract}

\section{Introduction}

Spiropyran (SP) is one of the very recent most studied photochromic molecules, which are popular materials in sunglasses, optical switching, optical data storage, nonlinear optical devices, optical nanoparticles, photonic crystal, and so on [1-21]. As is well known, the lowest electronic transition of SP occurs in the near UV region $(\lambda<400 \mathrm{~nm})$. As shown in Scheme 1, after photoexcitation the bond of Spiro-C and $\mathrm{O}$ atom is gradually broken and this compound converts to photoproducts called merocyanine MC. On the contrary, MC can also switch back to the closed form SP by irradiation in the visible region or heating.

The photochromic reactions of SP have been widely studied by time resolved spectroscopy to find the complex mechanism [22-28]. These experimental observations presume that the reaction would involve four steps. The first step corresponds to the initial excitation from the ground state to the excited state $\left({ }^{1} \pi \pi^{*}\right)$, which very soon (about $50 \mathrm{fs}$ ) evolves along the state $\left({ }^{1} \pi \sigma^{*}\right)$, this is the second step. Subsequently, the drastic changes in structures taking place on a time scale from subpico- to picosecond are the pyranic cycle opening and the $\mathrm{C}_{\text {spiro }}-\mathrm{O}$ bond cleavage. On the final step, a cis-cisoid open-form intermediate is firstly produced, and then several other planar MC photoproducts are formed by the geometrical transformation on pico- to nanosecond scale. Since the substituents on the SP and experimental conditions would be different, the formed products MCs and reaction mechanism are accordingly various. For instance, eight possible conformations for the MC would appear on the reaction process and can be distinguished from each other relative to three central bonds between $\mathrm{C}$ atoms (cis, $\mathrm{C}$, and trans, $\mathrm{T}$ ) as shown in Scheme 1. The most stable MC in solution is trans-trans-cis (TTC) isomer. In addition, very recent experiments show that the ring-opening reaction of unsubstituted SP $\left(1^{\prime}, 3^{\prime}, 3^{\prime}\right.$-trimethylspiro [2H-1-benzopyran$2,2^{\prime}$-indoline], also known as BIPS) [29] and double $\mathrm{NO}_{2}$ substituted derivative (6,8-dinitro-BIPS) [8] involve only singlet states, while a triple state makes a contribution to the dynamic of $\mathrm{NO}_{2}$-substituted SP (6-nitro-BIPS) [23]

The mechanisms of photochromic reactions of SP have also been studied by various high level quantum calculations 


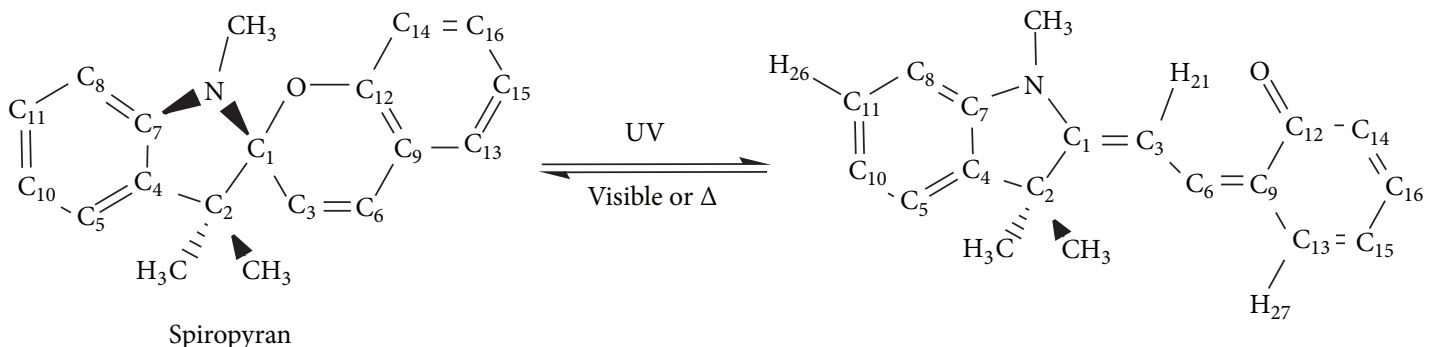

Merocyanine (MC, TTC)

Scheme 1: The scheme of reversible reaction between SP and MC.

TABLE 1: Key geometry parameters for the ground states of cis- and trans-SP forms as well as the corresponding mode compounds (bond distance in angstroms and dihedral angles in deg).

\begin{tabular}{llllccccc}
\hline Species & $\mathrm{C}_{1}-\mathrm{O}$ & $\mathrm{C}_{1}-\mathrm{N}$ & $\mathrm{C}_{1}-\mathrm{C}_{2}$ & $\mathrm{C}_{1}-\mathrm{C}_{3}$ & $\alpha$ & $\beta$ & $\gamma$ \\
\hline $\mathrm{mSPc}^{\mathrm{a}}$ & 1.428 & 1.465 & 1.540 & 1.515 & -97.8 & -1.0 & -11.6 & -9.3 \\
$\mathrm{mSPc}^{\mathrm{b}}$ & 1.445 & 1.452 & 1.544 & 1.502 & -98.9 & -3.6 & 177.1 \\
$\mathrm{mSPc}^{\mathrm{c}}$ & 1.422 & 1.449 & 1.542 & 1.506 & -101.6 & -1.6 & -9.5 & 176.5 \\
cis-SP $^{\mathrm{a}}$ & 1.385 & 1.456 & 1.605 & 1.530 & -101.7 & -2.8 & -8.9 & 174.2 \\
$\mathrm{mSPt}^{\mathrm{a}}$ & 1.413 & 1.442 & 1.570 & 1.536 & -141.8 & 2.9 & 8.0 & -175.3 \\
$\mathrm{mSPt}^{\mathrm{b}}$ & 1.448 & 1.447 & 1.551 & 1.502 & -142.6 & 3.2 & 9.3 & -177.2 \\
$\mathrm{mSPt}^{\mathrm{c}}$ & 1.424 & 1.443 & 1.548 & 1.507 & -142.6 & 1.9 & 10.2 & -177.8 \\
trans-SP $^{\mathrm{a}}$ & 1.404 & 1.435 & 1.613 & 1.511 & -148.7 & 5.5 & 8.6 & -180.1
\end{tabular}

${ }^{a}$ The present work; ${ }^{b}$ the previous work refers to [30] with CAM-B3LYP/6-31G(d) optimization; ${ }^{c}$ the previous work refers to [30] with CASSCF/6-31G(d) optimization.

using the model molecule [30-41]. There are three different pathways for the photochromic reaction proposed by computations. The first and second kinds of paths are both associated with the $\mathrm{C}_{\text {spiro }}-\mathrm{O}$ stretching and hydrogen-out-ofplane (HOOP) torsion modes (the $\mathrm{C}$ atom is connected by Spiro-C) of the excited SP [38-40]. The last pathway involves the lengthening $\mathrm{C}_{\text {spiro }}-\mathrm{N}$ bond and the internal conversion from the electronically excited states to the SP ground state through $\mathrm{CI}_{\mathrm{S} 1 / \mathrm{S} 0}(\mathrm{CN})$ [38-40].

In order to more directly interpret these mechanisms, the semiclassical dynamics of the photochemical reaction have been carried out by the real SP following the excitation using several laser pulses, which is necessary for the photoinduced chemical reaction $[42,43]$. The molecular dynamic method used in this work is called semiclassical electron-radiationion dynamics (SERID), on which the time-dependent quantum states are calculated for the valence electrons, while the radiation field and the motion of the nuclei are treated classically. The details of this method have been described elsewhere $[44,45]$. All degrees of freedom of real SP in the dynamic simulations are included in the calculations and the laser pulse is explicitly coupled to electrons. By monitoring the nuclear motions triggered by a specific laser pulse, it is possible to describe a realistic photochromic reaction path and provide complementary information for the clarification of the complex mechanisms of photochromic process of SP.

\section{Results and Discussion}

2.1. Optimized Ground-State Geometries. Two ground state geometries of SP, cis- and trans-SP conformers, respectively, are optimized within $5000 \mathrm{fs}$ dynamic simulation. In order to verify the geometry parameters, the mode compounds $\mathrm{mSPc}$ for cis- and mSPt for trans-isomers (in which three methyl groups are replaced by hydrogen) are also optimized. Hereafter, the primary dihedral angles $\mathrm{N}-\mathrm{C}_{1}-\mathrm{C}_{3}-\mathrm{C}_{6}, \mathrm{C}_{1}-\mathrm{C}_{3}-$ $\mathrm{C}_{6}-\mathrm{C}_{9}, \mathrm{C}_{3}-\mathrm{C}_{6}-\mathrm{C}_{9}-\mathrm{C}_{12}$, and $\mathrm{H}_{21}-\mathrm{C}_{3}-\mathrm{C}_{1}-\mathrm{C}_{6}$ (see Scheme 1) are, respectively, labeled as $\alpha, \beta, \gamma$, and $\tau$ that are consistent with the previous work $[39,40]$. The key geometry parameters for the ground state of cis- and trans-SP forms as well as the corresponding mode compounds are displayed in Table 1. It is clear that the molecular geometries for both $\mathrm{mSPc}$ and $\mathrm{mSPt}$ obtained from the present simulations are in well agreement with those from the previous DFT and CASSCF calculations. It is evident that the optimization from the present dynamic simulation could provide reasonable geometry parameters for the real cis- and trans-SP conformers. It should be mentioned that the remarkable lengthening of the $C_{1}-C_{2}$ bonds can be seen on the real cis- and trans-SP in comparison with the previous model SP, where the $\mathrm{C}_{2}$ atom is connected by two methyl groups not two $\mathrm{H}$ atoms on model compounds $\mathrm{mSPc}$ and mSPt. On the contrary, the $\mathrm{C}_{1}-\mathrm{O}$ bond (also labeled as $\mathrm{C}_{\text {spiro }}-\mathrm{O}$ above) is evidently shortened. The reason for the variations of these two bonds may arise from the donator character of methyl group, so that the electrons can be delocalized from $\mathrm{C}_{2}$ to an acceptor $\mathrm{O}$ atom.

2.2. Internal Conversion Process to the Ground State from Excited SP. In consistence with the experimental conditions of $316 \mathrm{~nm}$ on the gas phase [24] and nearby $350 \mathrm{~nm}$ in water [28], a laser pulse of $80 \mathrm{fs}$ (FWHM) with an effective photon 


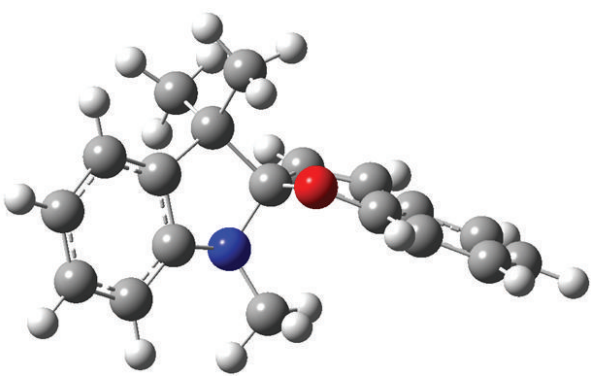

(a) $0 \mathrm{fs}$

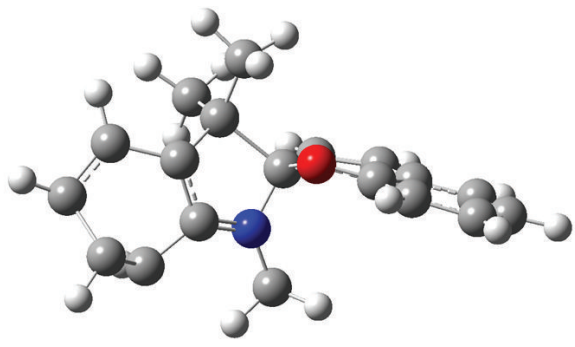

(c) $254 \mathrm{fs}$

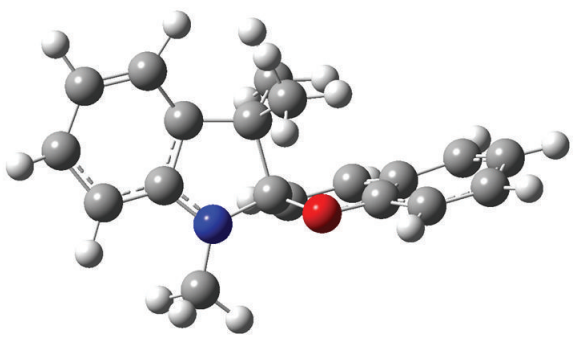

(e) $1658 \mathrm{fs}$

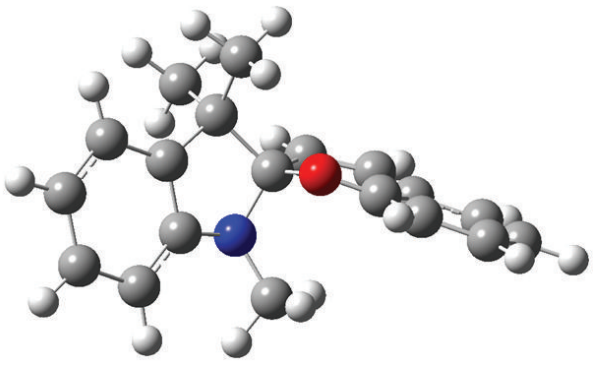

(b) $86 \mathrm{fs}$

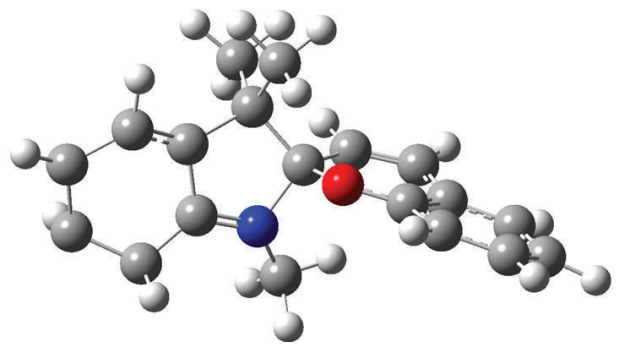

(d) $402 \mathrm{fs}$

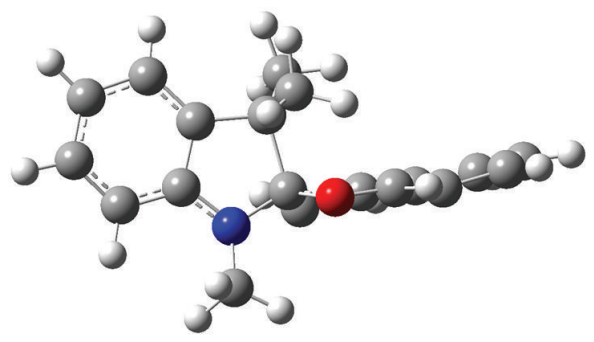

(f) $15000 \mathrm{fs}$

FIGURE 1: Snapshots taken at different times in a simulation of cis-SP in response to excitation by a $80 \mathrm{fs}$ (FWHM) laser pulse with a fluence of $0.188 \mathrm{~kJ} / \mathrm{m}^{2}$ and photon energy of $4.054 \mathrm{eV}$, Atom C, H, N, and O are, respectively, denoted by dark grey, French grey, blue, and red; (a)-(f) are at $0 \mathrm{fs}, 86 \mathrm{fs}, 254 \mathrm{fs}, 402 \mathrm{fs}, 1658 \mathrm{fs}$, and $15000 \mathrm{fs}$, respectively.

TABLE 2: Key geometry parameters for the dynamic simulation of internal conversion process of cis-SP (time in fs, bond distance in angstroms, and dihedral angles in deg).

\begin{tabular}{lcccccccrr}
\hline Time & $\mathrm{C}_{1}-\mathrm{O}$ & $\mathrm{C}_{1}-\mathrm{N}$ & $\mathrm{C}_{1}-\mathrm{C}_{2}$ & $\mathrm{C}_{1}-\mathrm{C}_{3}$ & $\alpha$ & $\beta$ & $\gamma$ & \multicolumn{1}{c}{$\tau$} \\
\hline 0 & 1.385 & 1.456 & 1.605 & 1.530 & -101.7 & -2.8 & -8.9 & -185.8 & -179.7 \\
86 & 1.441 & 1.458 & 1.570 & 1.524 & -99.8 & -3.7 & -9.1 & -188.9 & -183.0 \\
254 & 1.342 & 1.432 & 1.741 & 1.498 & -101.3 & -5.0 & -5.6 & -186.8 & -235.4 \\
402 & 1.466 & 1.513 & 1.538 & 1.486 & -98.1 & 0.6 & -12.5 & -179.3 & -245.7 \\
1658 & 1.411 & 1.455 & 1.664 & 1.539 & -169.1 & 18.4 & 4.1 & -156.8 & -158.7 \\
15000 & 1.326 & 1.475 & 1.676 & 1.523 & -164.1 & 25.7 & -10.0 & -134.1 & -199.7 \\
\hline
\end{tabular}

energy of $4.054 \mathrm{eV}$ (corresponding to $305.8 \mathrm{~nm}$ light) was applied to generate an excitation of cis-SP, in which the primary vibrations, particularly the $\mathrm{C}_{1}-\mathrm{C}_{2}$ stretching, are impressively activated. The photon energy selected corresponds to the energy difference between the HOMO-1 and LUMO+1 for the beginning ground-state geometry of the molecule in the present approximation. The simulations were run for various fluences and only one typical trajectory will be examined for a fluence of $0.188 \mathrm{~kJ} / \mathrm{m}^{2}$ that creates the desired vibration modes so that the radiationless deactivation of excited SP occurs.

Six snapshots from the simulation at different times and the critical geometry parameters of them are shown in Figure 1 and Table 2, respectively. Starting from the equilibrium geometry in the electronic ground state at $0 \mathrm{fs}$, the cis$\mathrm{SP}$ conformer is electronically excited by the laser pulse. The most impressive features of the nuclear motions of the excited molecule are the evident $\mathrm{C}_{1}-\mathrm{C}_{2}$ bond stretching, twist of $\alpha$, 
and HOOP torsion of the phenyl ring connected with $\mathrm{N}$ atom presented by the dihedral angle $\mathrm{H}_{26}-\mathrm{C}_{11}-\mathrm{C}_{8}-\mathrm{C}_{10}$ (labeled as $\theta)$.

The variations of dihedral angles $\alpha, \beta, \gamma, \tau$ with time are, respectively, plotted in Figure 2(a), the detailed changes about $\alpha, \tau, \theta$ in the very beginning of this simulation are also displayed in Figure 2(b) for illustration. It can be viewed that dihedral angle $\alpha$ twists largely from $-101.7^{\circ}$ at 0 fs to about $-160.0^{\circ}$ at about $10 \mathrm{ps}$, while both $\beta$ and $\gamma$ oscillate slightly about their initial value of $0^{\circ}$. This means that the initial cis$\mathrm{SP}$ can be transformed into trans-SP in $10 \mathrm{ps}$, since it can been seen in Table 1 that the dihedral angle $\alpha$ of stable cis- and trans-SP is $-97.8^{\circ}$ and $-148.7^{\circ}$, while $\beta$ and $\gamma$ are approximate to $0.0^{\circ}$. After that these angles mainly keep the values until the end of this simulation. It should be emphasized that the molecular vibration is started from the HOOP torsion of phenyl ring, corresponding to the change of dihedral angle $\theta$. It is obvious in Figure 2(b) that this angle oscillates and decreases from $-179.7^{\circ}$ at $0 \mathrm{fs}$ to $-254.5^{\circ}$ at $282 \mathrm{fs}$; after that the average value of $-180.0^{\circ}$ for $\theta$ is kept to the end and the variations of $\alpha$ and $\tau$ are initiate as shown in Figure 2(b) and Table 2. Similar to dihedral angle $\alpha$, the large torsion of $\tau$ is weakened at about $10 \mathrm{ps}$ and then the motion maintains an average value about $-160.0^{\circ}$. The bond stretches with time are not notable expert for the variation of $\mathrm{C}_{1}-\mathrm{C}_{2}$ bond. As shown in Figure 2(c), this bond is lengthened after about $210 \mathrm{fs}$ and then its value increases to $1.771 \AA$ at $248 \mathrm{fs}$. Subsequently, this bond oscillates largely and has a large value of more than $1.800 \AA$ around $4000 \mathrm{fs}$. After $10 \mathrm{ps}$, its length approximately decreases to the average value $1.630 \AA$ and keeps this value to the end.

The populations and energies of four interesting frontier molecular orbitals with time, including the HOMO-1, HOMO, LUMO, and LUMO+1, are shown in Figures 3(a) and 3(b), respectively. There are also a small fraction of electrons in the higher-lying unoccupied orbitals and a small fraction of holes in the lower-lying occupied orbitals. However, they do not play a notable role in the dynamical process discussed below. The variations of the electronic populations in different molecular orbitals induce the changes in the forces on the nuclei, driving them into motion. Many vibration modes of the molecule, including the dihedral motions, bond bending vibrations, and bond stretch vibrations, are excited. These excitations in turn change the gaps between the different molecular orbitals.

By the analysis of molecular orbital coefficients, the electronic transitions $\mathrm{HOMO}-1 \rightarrow$ LUMO, HOMO $-1 \rightarrow$ LUMO $+1, \quad \mathrm{HOMO} \rightarrow \mathrm{LUMO}$, and $\mathrm{HOMO} \rightarrow \mathrm{LUMO}+1$ of the stable cis-SP can, respectively, be denoted as $\pi_{H-1} \pi_{L}^{*}$, $\pi_{H-1} \pi_{L+1}^{*}, n_{H} \pi_{L}^{*}$, and $n_{H} \pi_{L+1}^{*}$ at $0 \mathrm{fs}$. With the photon excitation, the electronic transitions vary with time. Before $86 \mathrm{fs}$, it can be seen in Figure 3(a) that main electronic transition is from HOMO-1 to LUMO+1, corresponding to $\pi_{H-1} \pi_{L+1}^{*}$. At $86 \mathrm{fs}$, it is distinct in Figure $4(\mathrm{~b})$ that the HOMO and HOMO-1 are degenerate and then the significant electronic transfer changes from $\mathrm{HOMO}-1 \rightarrow$ LUMO $\left(\pi_{H-1} \pi_{L}^{*}\right)$ to HOMO $\rightarrow$ LUMO $\left(\pi_{H} \pi_{L}^{*}\right)$. The dominate transition of $\pi_{H} \sigma_{L}^{*}$ is at $254 \mathrm{fs}$, corresponding to the degeneracy of HOMO and LUMO. The main nuclear
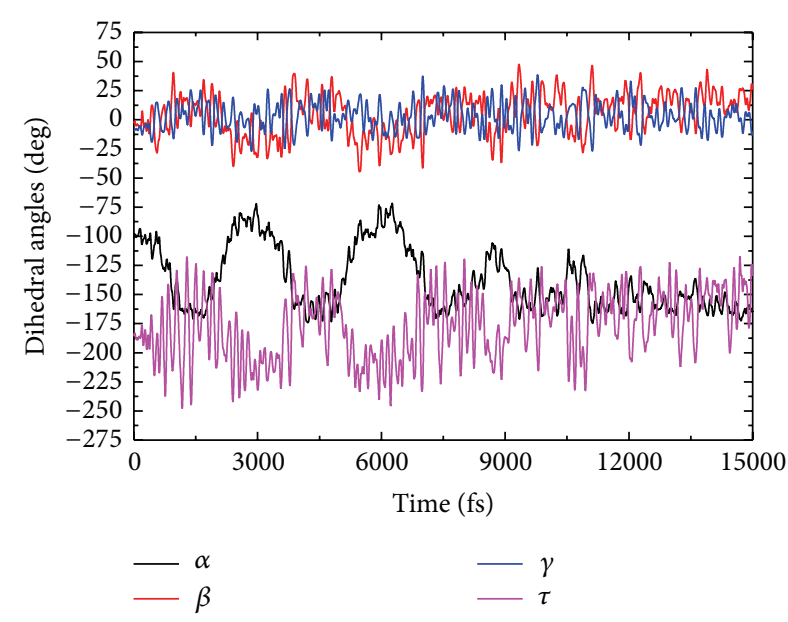

(a)

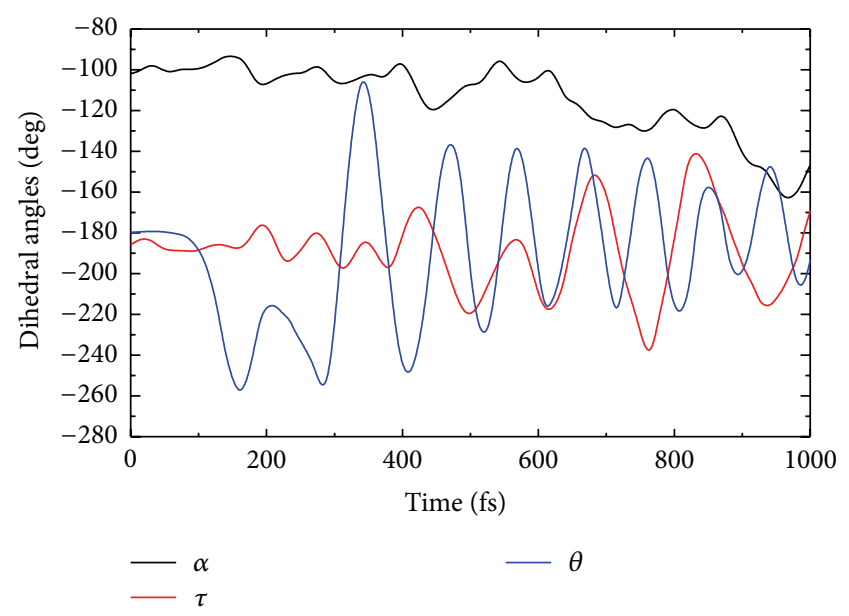

(b)

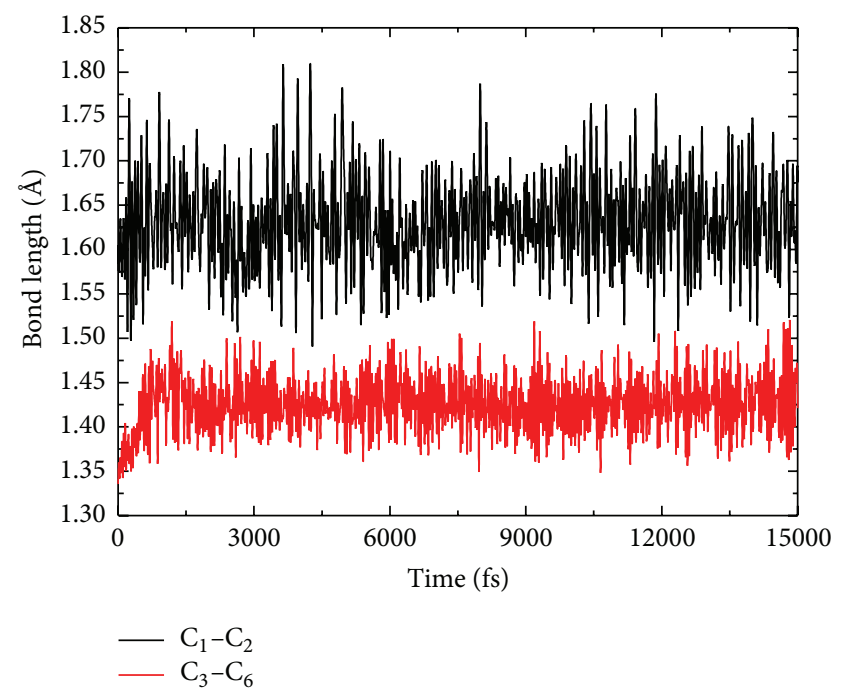

(c)

Figure 2: Changes in (a) the dihedral angles $\alpha, \beta, \gamma$, and $\tau$; (b) $\alpha, \tau$, and $\theta$; (c) $\mathrm{C}_{1}-\mathrm{C}_{2}$ and $\mathrm{C}_{3}-\mathrm{C}_{6}$ bond lengthens of cis-SP subjected excitation by a $80 \mathrm{fs}$ (FWHM) laser pulse with a fluence of $0.188 \mathrm{~kJ} / \mathrm{m}^{2}$ and photon energy of $4.054 \mathrm{eV}$. 
TABLE 3: Key geometry parameters for the dynamic simulation of photochromic ring-opening reaction of trans-SP (time in fs, bond distance in angstroms, and dihedral angles in deg) [41].

\begin{tabular}{lccccccccc}
\hline Time & $\mathrm{C}_{1}-\mathrm{O}$ & $\mathrm{C}_{1}-\mathrm{N}$ & $\mathrm{C}_{1}-\mathrm{C}_{2}$ & $\mathrm{C}_{1}-\mathrm{C}_{3}$ & $\alpha$ & $\beta$ & $\gamma$ & \multicolumn{1}{c}{$\tau$} \\
\hline 0 & 1.404 & 1.435 & 1.613 & 1.511 & -148.7 & 5.5 & 8.6 & 179.9 & 181.8 \\
244 & 1.465 & 1.432 & 1.620 & 1.519 & -154.4 & 1.0 & 20.1 & 163.0 & 244.1 \\
422 & 1.571 & 1.430 & 1.618 & 1.556 & -181.1 & 45.8 & -1.0 & 134.0 & 142.7 \\
616 & 3.618 & 1.350 & 1.607 & 1.504 & -87.4 & -10.8 & 58.6 & 203.7 & 150.8 \\
4848 & 3.945 & 1.380 & 1.530 & 1.444 & -201.6 & -179.6 & -37.7 & 190.2 & 190.8 \\
15000 & 4.400 & 1.371 & 1.527 & 1.450 & -160.3 & -182.8 & 39.7 & 175.1 & 189.7 \\
\hline
\end{tabular}

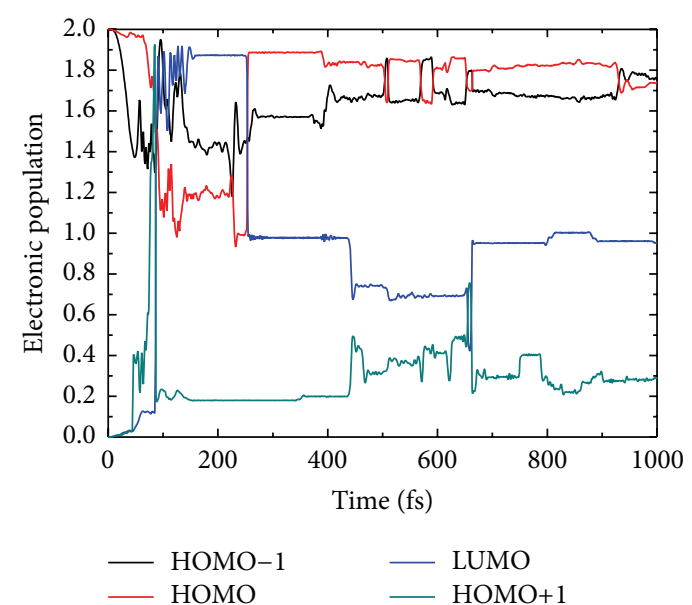

(a)

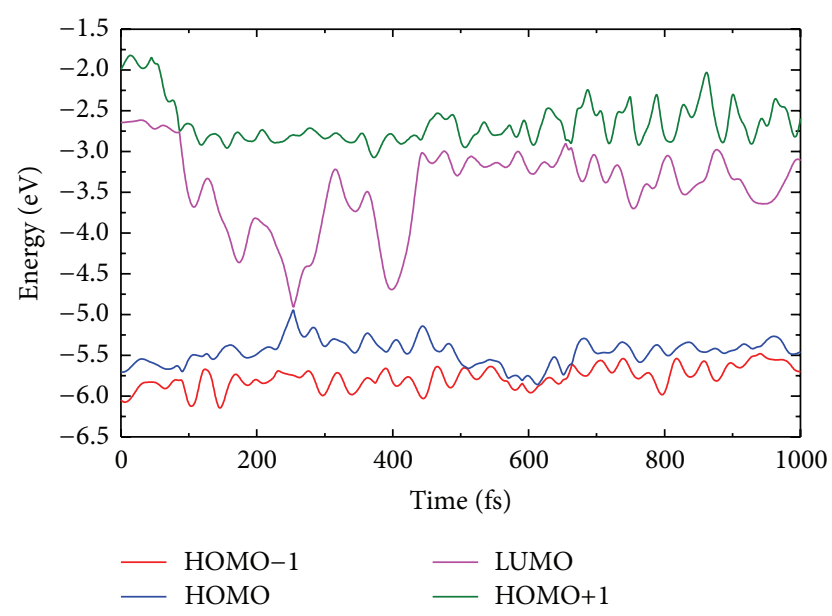

(b)

FIGURE 3: Variations with time of (a) the electronic populations and (b) energies of the HOMO-1, HOMO, LUMO, and LUMO+1 orbitals of cis-SP following application of a $80 \mathrm{fs}$ (FWHM) laser pulse with a fluence of $0.188 \mathrm{~kJ} / \mathrm{m}^{2}$ and photon energy of $4.054 \mathrm{eV}$.

motion before $254 \mathrm{fs}$ can be found to be the HOOP torsion of phenyl group nearby $\mathrm{N}$ atom as described in Figure 2(b). This torsion is similar to those on one pathway of radiationless deactivation reaction of $9 \mathrm{H}$-adenine as reported by Lei et al. [46], where there is an out-of-plane twist of the $\mathrm{C}_{2}-\mathrm{H}$ bond of pyrimidine ring before the significant transition between HOMO and LUMO. After that the excited electrons radiationless decay back to the ground state and no remarkable electron transfer between occupied and unoccupied orbitals exists until the end of this simulation. Accordingly, the obtained kinetic energies result in the large variations of dihedral angles $\alpha$, and bond $\mathrm{C}_{1}-\mathrm{C}_{2}$ as mentioned above.

With these simulation results, it is easy to study the mechanism of internal conversion process from excited SP to the ground state. In fact, this process corresponds to the photoisomerization from cis-SP to trans-SP. This is an ultrafast process with excited state lifetime only $254 \mathrm{fs}$. The main nuclear motions on the excited states are the HOOP torsion leading to the deformation of phenyl group nearby $\mathrm{N}$ atom, while the isomerization takes place after the electron transition from $S_{1}$ to $S_{0}$ and go along the latter potential energy surface.

2.3. Photochromic Ring-Opening Reaction of SP. As reported in preceding paper [47] and redescribed in Figure 4 and Table 3, the representative trajectory for the phototriggered ring-opening process shows that ring-opening reaction of excited SP starts from the cooperative HOOP torsions on the excited states and the lifetime of excited states is $422 \mathrm{fs}$. Then on the ground state the $\mathrm{C}_{1}-\mathrm{O}$ bond is broken and the MCTCC is formed before $616 \mathrm{fs}$; after that a transient MC-CCC isomer appears and is suddenly replaced by the former MC conformation until $4848 \mathrm{fs}$. After that the final stable TTC form of MC is created and kept up to the end. This means that the ring-opening process takes about $616 \mathrm{fs}$, which basically agrees with $900 \mathrm{fs}$ measured by the experiments on the gas phase [24]. The subsequent isomerization on the ground state takes about 4 ps, which is less than about 20 ps obtained by the previous studies $[25,31]$. The expected mechanism for photochromic ring-opening here is also suggested by Liu and Morokuma in more accurate theoretical computations $[39,40]$.

2.4. Mechanism Differences between Real and Model SP. Except for the photochromic processes of SP described in Sections 2.2 and 2.3, there are many other trajectories run in this work; most of them provide information for the ionization of SP molecule, according to the $\mathrm{C}_{1}-\mathrm{C}_{2}$ dissociation. For instance, the lengthening of $\mathrm{C}_{1}-\mathrm{C}_{2}$ bond of one representative trajectory for the excited SP is presented in Figure 5. The lifetime of excited state on this trajectory is evaluated to be about $254 \mathrm{fs}$, while this bond is completely 


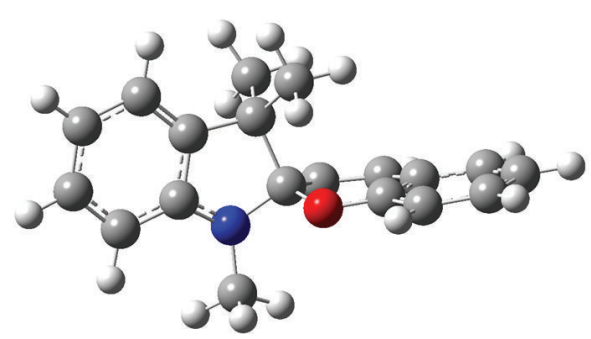

(a) $0 \mathrm{fs}$

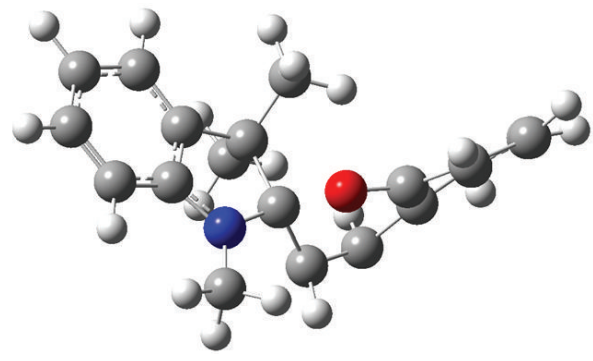

(c) $422 \mathrm{fs}$

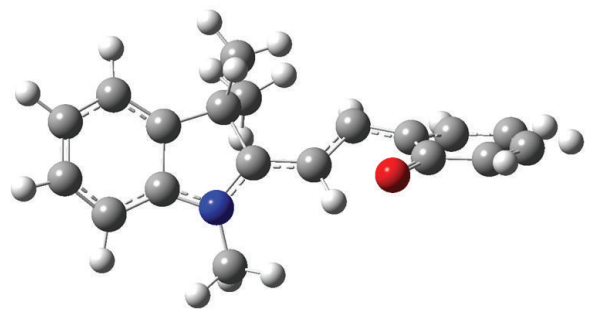

(e) $4848 \mathrm{fs}$

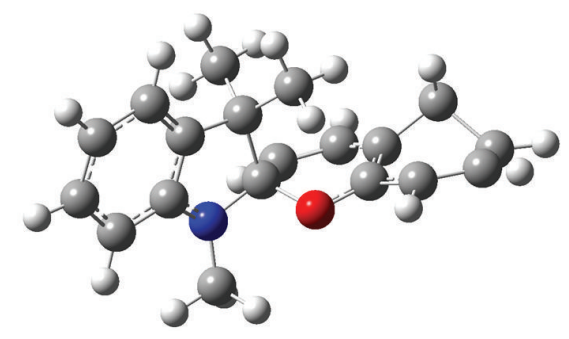

(b) $244 \mathrm{fs}$

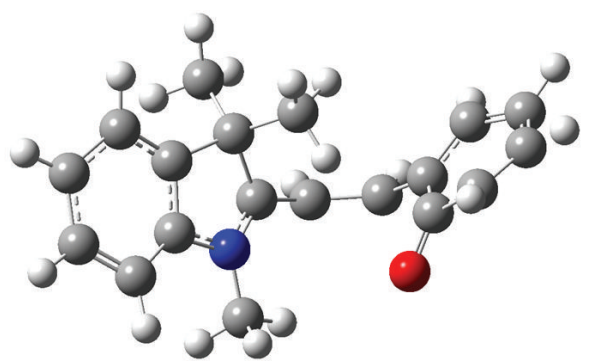

(d) $616 \mathrm{fs}$

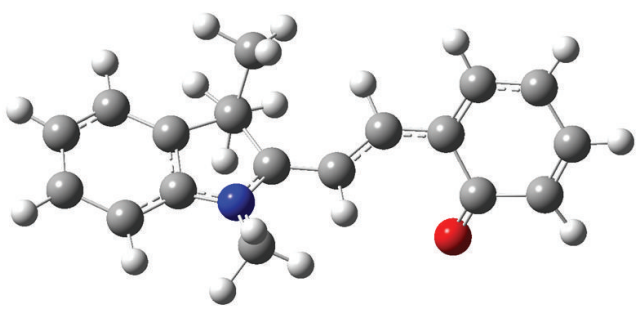

(f) $15000 \mathrm{fs}$

FIGURE 4: Snapshots taken at different times in a simulation of trans-SP in response to excitation by a $80 \mathrm{fs}$ (FWHM) laser pulse with a fluence of $0.139 \mathrm{~kJ} / \mathrm{m}^{2}$ and photon energy of $3.972 \mathrm{eV}$, Atom $\mathrm{C}, \mathrm{H}, \mathrm{N}$, and $\mathrm{O}$ are, respectively, denoted by dark grey, French grey, blue, and red; (a)-(f) are at $0 \mathrm{fs}, 244 \mathrm{fs}, 422 \mathrm{fs}, 616 \mathrm{fs}, 4848 \mathrm{fs}$, and $15000 \mathrm{fs}$, respectively [41].

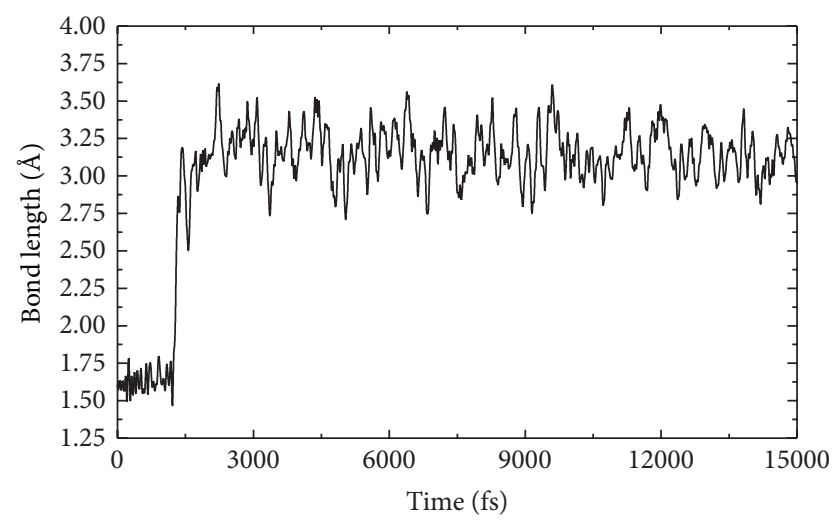

FIGURE 5: Variations with time of $\mathrm{C}_{1}-\mathrm{C}_{2}$ bond of cis-SP following application of a $80 \mathrm{fs}$ (FWHM) laser pulse with a fluence of $0.186 \mathrm{~kJ} / \mathrm{m}^{2}$ and photon energy of $4.054 \mathrm{eV}$ and excited state lifetime of $254 \mathrm{fs}$.

broken after about $1430 \mathrm{fs}$ when the bond length approximately maintains a large average value. The dissociated $\mathrm{C}_{1}-$ $\mathrm{C}_{2}$ bond is not recovered on this simulation as the high kinetic energies on the ground state are not dispersed to other vibration modes of SP. In comparison with the pathways in the above sections, it can be found the ionization and isomerization of excited SP with the same lifetime $254 \mathrm{fs}$ are both more ultrafast than the ring-opening reaction with lifetime $422 \mathrm{fs}$, which confirm the previous conclusion that the quantum yield of MC products of this photochromic reaction is relatively less, since the internal conversion from the excited state to the ground state of SP is even more efficient $[24,28]$.

For the model compounds of SP, the pathways are of a wide variety. With photon excitations, all the four bonds between the central spiro- $\mathrm{C}$ and its connected atoms would be dissociated, since the bond lengths of them are similar. The most possible bond lengthening take places between $\mathrm{C}_{1}$ and $\mathrm{C}_{2}$ bond in consistent with the real SP molecule. All the typical simulation trajectories of these paths with the photoexcitations are displayed on the Supporting Information from Figures S1 to S7 available online at http://dx.doi.org/10.1155/2014/541791, corresponding to the cleavage of $\mathrm{C}_{1}-\mathrm{O}$ bond in Figure S1, the cleavage and then recovery of $\mathrm{C}_{1}-\mathrm{C}_{3}$ bond in Figure $\mathrm{S} 2$, the dissociations of $\mathrm{C}_{1}-\mathrm{N}, \mathrm{C}_{1}-\mathrm{C}_{2}$, and $\mathrm{C}_{1}-\mathrm{C}_{3}$ bonds in Figures $\mathrm{S} 3-\mathrm{S} 5$, the isomerizations of $\mathrm{mSPc}$ and $\mathrm{mSPt}$ in Figures $\mathrm{S} 6$ and $\mathrm{S}$, 
respectively. Among these trajectories, it is distinct in Figure S1 that, through electronic transition from the excited state to the ground state at $560 \mathrm{fs}$, the product MC-CCC, which is measured by the experiment but assumed by the computation, is formed. At about $12 \mathrm{ps,} \mathrm{this} \mathrm{MC}$ conformation is started to convert to the more stable product MC-CTC accompanied with the lengthening of $\mathrm{C}_{1}-\mathrm{O}$ bond until the final MC-CTC is completely formed after about 14 ps. This simulation provides a direct evidence for the very recent mechanism proposed by Liu and Morokuma $[39,40]$ and the previous findings that the transformation from unstable MC form to the final MC conformation takes about 20 ps [25, 31]. In addition, it can be found in Figure S2 that the dissociation of $\mathrm{C}_{1}-\mathrm{C}_{3}$ bond can be recovered at $13411 \mathrm{fs}$ from the broken form at $9894 \mathrm{fs}$ on the typical trajectory, accompanied with the recovery of cis-SP as the $\alpha, \beta$, and $\gamma$ finally twist back to their initial values, which is similar to the previous suggested process that dissociation of $\mathrm{C}_{1}-\mathrm{N}$ bond has also a chance back to the original bonding form $[39,40]$.

The reason for only three types of trajectories on the simulations of the real SP may be partially deduced by the bond distances of these four bonds around the central spiroC. As described on Table 1; no matter cis- or trans-SP, the $\mathrm{C}_{1}-\mathrm{C}_{2}$ bond of the real molecule is elongated while other bonds are shortened in comparison with the model ones, so that the break of $\mathrm{C}_{1}-\mathrm{C}_{2}$ bond is easier than other bonds. On the other hand, the $\mathrm{C}_{1}-\mathrm{O}$ bond can be lengthened since it points to the stable MC product, while the dissociation of $\mathrm{C}_{1}-\mathrm{N}$ or $\mathrm{C}_{1}-\mathrm{C}_{3}$ would result in the ionization of SP, which is more active than both SP and MC stable structures. Hence, no dissociation of $\mathrm{C}_{1}-\mathrm{N}$ or $\mathrm{C}_{1}-\mathrm{C}_{3}$ can be discovered on the dynamic simulations of the real SP, which is different from the previous conclusions that the internal conversion occurs accompanying the dissociation of $\mathrm{C}_{1}-\mathrm{N}$ bond from the model compounds [38-40].

\section{Conclusions}

In summary, we have investigated the photochromic reaction mechanism of SP, employing realistic simulation approach that couples the dynamics of electrons and nuclei. The main simulations follow two different excitations that lead to two altered reaction paths: one is the ultrafast photoisomerization from cis-SP to trans-SP, with respect to internal conversion process to the ground state from excited SP. The process on the excited state involves HOOP torsion of phenyl ring nearby $\mathrm{N}$ atom in $254 \mathrm{fs}$, while the isomerization takes place after the electron transition $\pi_{H} \sigma_{L}^{*}$ to $S_{0}$ and goes along the latter potential energy surface with the transformation from cis- to trans-SP in about 10 ps. The other path corresponds to the ring-opening reaction of trans-SP, with regard to the SP to the stable MC conformation MC-TTC. Similarly, the process on the excited state with the lifetime $422 \mathrm{fs}$ involves two cooperative HOOP torsions of two connected six membered rings. After the electronic transition $\pi_{H} \sigma_{L}^{*}$ to the ground state, the first MC product MC-TCC is formed and $\mathrm{C}_{1}-\mathrm{O}$ is completely broken after $616 \mathrm{fs}$, at this moment the unstable product MC-CCC, assigned to appear shortly after photoexcitation $[48,49]$, emerges and rapidly converts back to the origin MC product MC-TCC. After $4848 \mathrm{fs}$, this product is transformed to the final stable $\mathrm{MC}$ conformation MC-TTC with the large twist around the central $\mathrm{C}_{3}-\mathrm{C}_{6}$ bond. This simulation trajectory provides a direct proof for confirming the proposed mechanism by the very recent computations $[39,40]$.

Unlike the possible photodissociation of all the four bonds around the central $\mathrm{C}_{1}$ atom of the model SP, containing the previously mentioned $\mathrm{C}_{1}-\mathrm{N}$ bond cleavage [38-40], the real molecule would usually be ionized with only the $\mathrm{C}_{1}-$ $\mathrm{C}_{2}$ dissociation. The ultrafast process of the photoinduced ionization and isomerization of SP plays very important roles in the effective internal conversion and less quantum yields of MC products after the photon excitations, which agrees with the previously experimental observations [24, 28].

The current dynamic simulations provide more important complementarity for the mechanism of the ultrafast photochromic ring-opening reaction as well as the internal conversion process of SP. The findings here confirm the previously theoretical proposals for the explanations of the ultrafast processes and even supply other possible reaction paths, for instance, the ultrafast photoisomerization from cisSP to trans-SP. The foundational information shown in this paper would be available to interpret experimental conservations and helpful to design new photochromic devices based on these kinds of compounds.

\section{Conflict of Interests}

The authors declare that there is no conflict of interests regarding the publication of this paper.

\section{Acknowledgments}

This work is supported by the National Natural Science Foundation of China (nos. 21003100, 21073242), Natural Science Basic Research Plan in Shaanxi Province of China (nos. 2011JQ2013 and 2014JQ2060), and Special Fund of Education Department of Shaanxi Province (no. 12JK0619). The authors also thank Professor Zhenyi Wen, Associate Professor Huixian Han, and Bingbing Suo for the helpful suggestions.

\section{References}

[1] B. L. Feringa, Molecular Switches, Wiley-VCH, Weinheim, Germany, 2001.

[2] H. Dürr and H. Bouas-Laurent, Photochromism: Molecules and Systems, Elsevier, Amsterdam, The Netherlands, 2003.

[3] H. Bouas-Laurent and H. Dürr, "Organic photochromism," Pure and Applied Chemistry, vol. 73, no. 4, pp. 639-665, 2001.

[4] M. Heilemann, P. Dedecker, J. Hofkens, and M. Sauer, "Photoswitches: key molecules for subdiffraction-resolution fluorescence imaging and molecular quantification," Laser and Photonics Reviews, vol. 3, no. 1-2, pp. 180-202, 2009.

[5] R. C. Bertelson, "Reminiscences about organic photochromics," Molecular Crystals and Liquid Crystals Science and Technology A: Molecular Crystals and Liquid Crystals, vol. 246, pp. 1-8, 1994. 
[6] J. P. Desvergne, H. Bouas-Laurent, and A. Deffieux, "Photochromism of anthracene derivatives in fluid solutions and polymers," Molecular Crystals and Liquid Crystals Science and Technology A: Molecular Crystals and Liquid Crystals, vol. 246, pp. 111-118, 1994.

[7] K. Schaffner, R. Schmidt, and H. D. Brauer, "Photochromism based on the reversible reaction of singlet oxygen with aromatic compounds," Molecular Crystals and Liquid Crystals Science and Technology A: Molecular Crystals and Liquid Crystals, vol. 246, pp. 119-125, 1994.

[8] J. Buback, M. Kullmann, F. Langhojer et al., "Ultrafast bidirectional photoswitching of a spiropyran," Journal of the American Chemical Society, vol. 132, no. 46, pp. 16510-16519, 2010.

[9] G. Berkovic, V. Krongauz, and V. Weiss, "Spiropyrans and spirooxazines for memories and switches," Chemical Reviews, vol. 100, no. 5, pp. 1741-1753, 2000.

[10] D. A. Parthenopoulos and P. M. Rentzepis, “Three-dimensional optical storage memory," Science, vol. 245 , no. 4920 , pp. $843-$ 845, 1989.

[11] B. L. Feringa, R. A. van Delden, N. Koumura, and E. M. Geertsema, "Chiroptical molecular switches," Chemical Reviews, vol. 100, no. 5, pp. 1789-1816, 2000.

[12] F. M. Raymo and S. Giordani, "All-optical processing with molecular switches," Proceedings of the National Academy of Sciences of the United States of America, vol. 99, no. 8, pp. 49414944, 2002.

[13] K. Fujimoto, M. Amano, Y. Horibe, and M. Inouye, "Reversible photoregulation of helical structures in short peptides under indoor lighting/dark conditions," Organic Letters, vol. 8, no. 2, pp. 285-287, 2006.

[14] W. Zhou, D. Chen, J. Li et al., "Photoisomerization of spiropyran for driving a molecular shuttle," Organic Letters, vol. 9, no. 20, pp. 3929-3932, 2007.

[15] S. Silvi, A. Arduini, A. Pochini et al., "A simple molecular machine operated by photoinduced proton transfer," Journal of the American Chemical Society, vol. 129, no. 44, pp.13378-13379, 2007.

[16] D. S. Achilleos, T. A. Hatton, and M. Vamvakaki, "Light-regulated supramolecular engineering of polymeric nanocapsules," Journal of the American Chemical Society, vol. 134, no. 13, pp. 5726-5729, 2012.

[17] J. Chen, P. Zhang, G. Fang et al., "Synthesis and characterization of novel reversible photoswitchable fluorescent polymeric nanoparticles via one-step miniemulsion polymerization," Journal of Physical Chemistry B, vol. 115, no. 13, pp. 3354-3362, 2011.

[18] E. A. Osborne, B. R. Jarrett, C. Tu, and A. Y. Louie, "Modulation of $\mathrm{T}_{2}$ relaxation time by light-induced, reversible aggregation of magnetic nanoparticles," Journal of the American Chemical Society, vol. 132, no. 17, pp. 5934-5935, 2010.

[19] M. K. Maurer, I. K. Lednev, and S. A. Asher, "Photoswitchable spirobenzopyran-based photochemically controlled photonic crystals," Advanced Functional Materials, vol. 15, no. 9, pp. 14011406, 2005.

[20] R. Q. Albuquerque, J. Kühni, P. Belser, and L. de Cola, “On the reversible photoisomerization of spiropyran-modified zeolite $\mathrm{L}$ single crystals," A European Journal of Chemical Physics and Physical Chemistry, vol. 11, no. 3, pp. 575-578, 2010.

[21] T. A. Darwish, R. A. Evans, M. James, N. Malic, G. Triani, and T. L. Hanley, " $\mathrm{CO}_{2}$ triggering and controlling orthogonally multiresponsive photochromic systems," Journal of the American Chemical Society, vol. 132, no. 31, pp. 10748-10755, 2010.
[22] N. Tamai and H. Miyasaka, "Ultrafast dynamics of photochromic systems," Chemical Reviews, vol. 100, no. 5, pp. 1875$1890,2000$.

[23] C. Lenoble and R. S. Becker, "Photophysics, photochemistry, kinetics, and mechanism of the photochromism of $6^{\prime}$ nitroindolinospiropyran," Journal of Physical Chemistry, vol. 90, no. 1, pp. 62-65, 1986.

[24] M. Rini, A. K. Holm, E. T. J. Nibbering, and H. Fidder, "Ultrafast UV-mid-IR investigation of the ring opening reaction of a photochromic spiropyran," Journal of the American Chemical Society, vol. 125, no. 10, pp. 3028-3034, 2003.

[25] L. Poisson, K. D. Raffael, B. Soep, J.-M. Mestdagh, and G. Buntinx, "Gas-phase dynamics of spiropyran and spirooxazine molecules," Journal of the American Chemical Society, vol. 128, no. 10, pp. 3169-3178, 2006.

[26] M. Kullmann, S. Ruetzel, J. Buback, P. Nuernberger, and T. Brixner, "Reaction dynamics of a molecular switch unveiled by coherent two-dimensional electronic spectroscopy," Journal of the American Chemical Society, vol. 133, no. 33, pp. 13074-13080, 2011.

[27] J. Hobley, U. Pfeifer-Fukumura, M. Bletz, T. Asahi, H. Masuhara, and H. Fukumura, "Ultrafast photo-dynamics of a reversible photochromic spiropyran," Journal of Physical Chemistry A, vol. 106, no. 10, pp. 2265-2270, 2002.

[28] J. Kohl-Landgraf, M. Braun, C. Özçoban, D. P. N. Gonçalves, A. Heckel, and J. Wachtveitl, "Ultrafast dynamics of a spiropyran in water," Journal of the American Chemical Society, vol. 134, no. 34, pp. 14070-14077, 2012.

[29] N. P. Ernsting and T. Arthen-Engeland, "Photochemical ringopening reaction of indolinospiropyrans studied by subpicosecond transient absorption," Journal of Physical Chemistry, vol. 95, no. 14, pp. 5502-5509, 1991.

[30] P. Celani, F. Bernardi, M. Olivucci, and M. A. Robb, "Conical intersection mechanism for photochemical ring opening in benzospiropyran compounds," Journal of the American Chemical Society, vol. 119, no. 44, pp. 10815-10820, 1997.

[31] Y. Sheng, J. Leszczynski, A. A. Garcia, R. Rosario, D. Gust, and J. Springer, "Comprehensive theoretical study of the conversion reactions of spiropyrans: substituent and solvent effects," Journal of Physical Chemistry B, vol. 108, no. 41, pp. 16233-16243, 2004.

[32] B. O. Roos, Advances in Chemical Physics, vol. 69, John Wiley \& Sons, New York, NY, USA, 1987.

[33] J. Finley, P.-Å. Malmqvist, B. O. Roos, and L. Serrano-Andrés, "The multi-state CASPT2 method," Chemical Physics Letters, vol. 288, no. 2-4, pp. 299-306, 1998.

[34] Y. Kurashige and T. Yanai, "High-performance ab initio density matrix renormalization group method: applicability to largescale multireference problems for metal compounds," Journal of Chemical Physics, vol. 130, no. 23, Article ID 234114, 2009.

[35] D. Ghosh, J. Hachmann, T. Yanai, and G. K. L. Chan, “Orbital optimization in the density matrix renormalization group, with applications to polyenes and $B$-carotene," Journal of Chemical Physics, vol. 128, no. 14, Article ID 144117, 2008.

[36] Y. Kurashige and T. Yanai, "Second-order perturbation theory with a density matrix renormalization group self-consistent field reference function: theory and application to the study of chromium dimer," Journal of Chemical Physics, vol. 135, no. 9, Article ID 094104, 2011.

[37] I. Gómez, M. Reguero, and M. A. Robb, "Efficient photochemical merocyanine-to-spiropyran ring closure mechanism 
through an extended conical intersection seam. A model CASSCF/CASPT2 study," Journal of Physical Chemistry A, vol. 110, no. 11, pp. 3986-3991, 2006.

[38] S.-L. Marta, E. Carlos Manuel, H.-R. Jose, and S.-A. Luis, "Ultrafast ring-opening/closing and deactivation channels for a model spiropyran-merocyanine system," Journal of Physical Chemistry A, vol. 115, no. 33, pp. 9128-9138, 2011.

[39] F. Liu and K. Morokuma, "Multiple pathways for the primary step of the spiropyran photochromic reaction: a CASPT2//CASSCF study," Journal of the American Chemical Society, vol. 135, no. 29, pp. 10693-10702, 2013.

[40] F. Y. Liu, Y. Kurashige, T. Yanai, and K. Morokuma, "Multireference $\mathrm{Ab}$ initio density matrix renormalization group (DMRG)CASSCF and DMRG-CASPT2 Study on the Photochromic Ring Opening of Spiropyran," Journal of Chemical Theory and Computation, vol. 9, no. 10, pp. 4462-4469, 2013.

[41] V. I. Minkin, A. V. Metelitsa, I. V. Dorogan, B. S. Lukyanov, S. O. Besugliy, and J.-C. Micheau, "Spectroscopic and theoretical evidence for the elusive intermediate of the photoinitiated and thermal rearrangements of photochromic spiropyrans," Journal of Physical Chemistry A, vol. 109, no. 42, pp. 9605-9616, 2005.

[42] T.-S. Chu, Y. Zhang, and K.-L. Han, "The time-dependent quantum wave packet approach to the electronically nonadiabatic processes in chemical reactions," International Reviews in Physical Chemistry, vol. 25, no. 1-2, pp. 201-235, 2006.

[43] K. Han, J. Huang, S. Chai, S. Wen, and W. Deng, "Anisotropic mobilities in organic semiconductors," Nature Protocol Exchange, 2013.

[44] Y. Dou, B. R. Torralva, and R. E. Allen, "Semiclassical electronradiation-ion dynamics (SERID) and cis-trans photoisomerization of butadiene," Journal of Modern Optics, vol. 50, no. 15-17, pp. 2615-2643, 2003.

[45] Y. Dou and R. E. Allen, "Another important coordinate in the photoisomerization of cis-stilbene," Chemical Physics Letters, vol. 378, no. 3-4, pp. 323-329, 2003.

[46] Y. Lei, S. Yuan, Y. Dou, Y. Wang, and Z. Wen, "Detailed dynamics of the nonradiative deactivation of adenine: a semiclassical dynamics study," Journal of Physical Chemistry A, vol. 112, no. 37, pp. 8497-8504, 2008.

[47] G. Zhai, P. Yang, S. Wu, Y. Lei, and Y. Dou, "A semiclassical molecular dynamics of the photochromic ring-opening reaction of spiropyran," Chinese Chemical Letters, vol. 25, no. 5, pp. 727-731, 2014.

[48] S. A. Krysanov and M. V. Alfimov, "Ultrafast formation of transients in spiropyran photochromism," Chemical Physics Letters, vol. 91, no. 1, pp. 77-80, 1982.

[49] S. A. Antipin, A. N. Petrukhin, F. E. Gostev et al., "Femtosecond transient absorption spectroscopy of non-substituted photochromic spirocompounds," Chemical Physics Letters, vol. 331 , no. 5-6, pp. 378-386, 2000. 

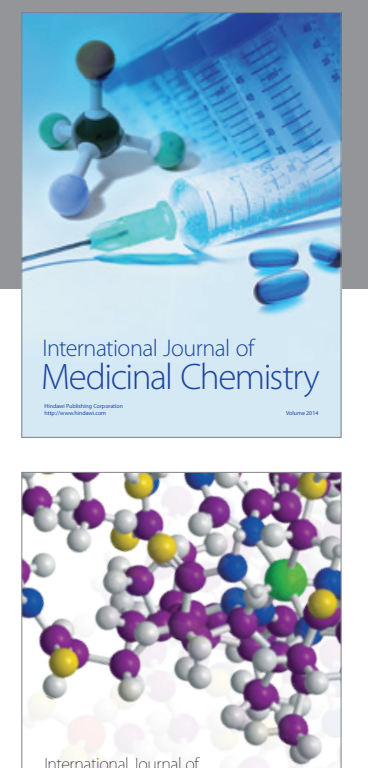

\section{Carbohydrate} Chemistry

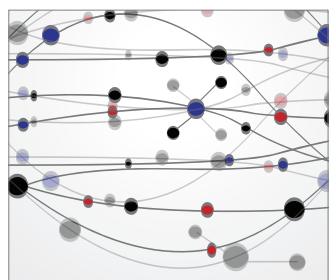

The Scientific World Journal
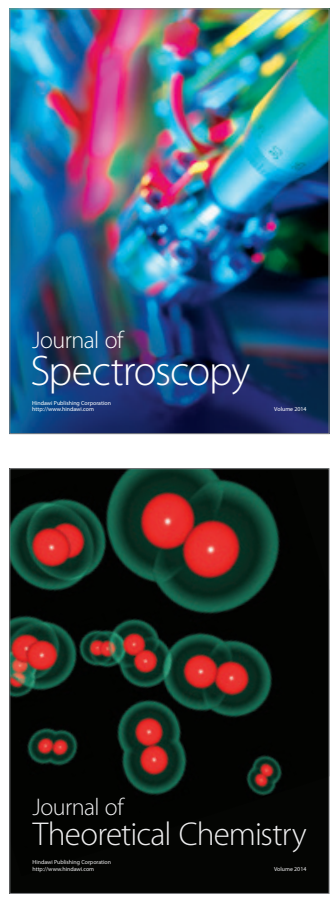
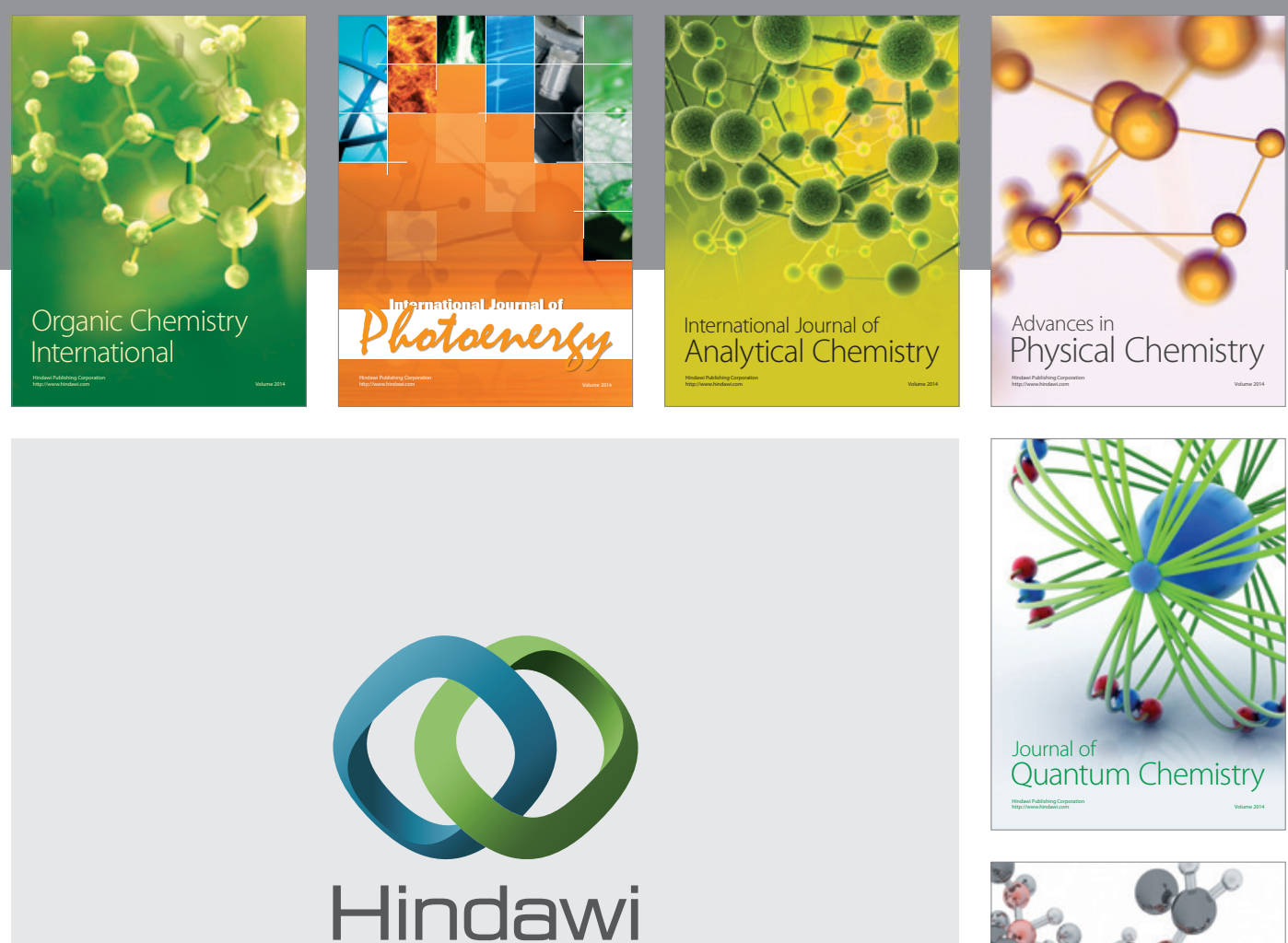

Submit your manuscripts at

http://www.hindawi.com

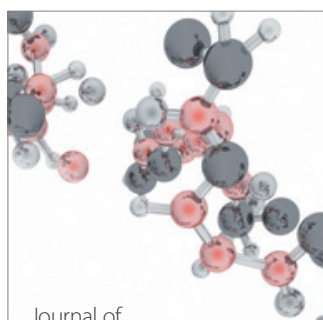

Analytical Methods

in Chemistry

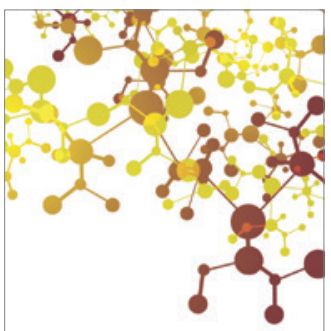

Journal of

Applied Chemistry

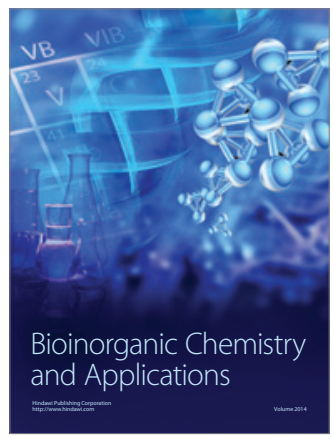

Inorganic Chemistry
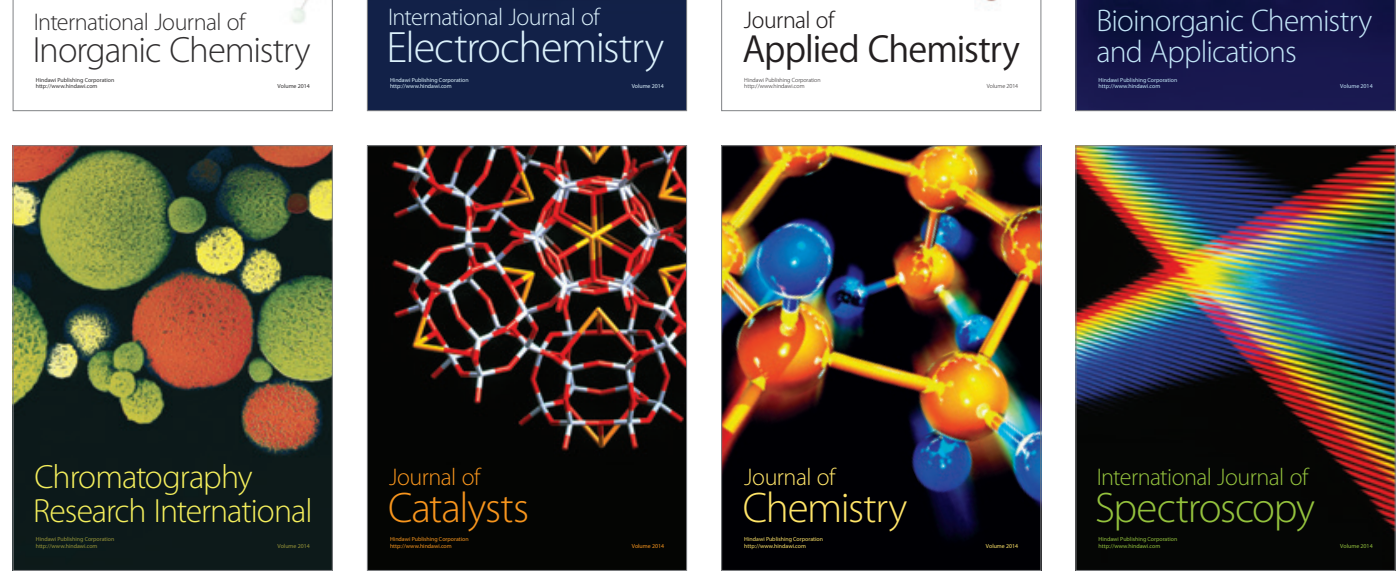